BI-TP 98/31

September 1998

\title{
Lattice Gravity and Random Surfaces
}

\author{
Gudmar Thorleifsson \\ Facultät für Physik, Universität Bielefeld D-33615, Bielefeld, Germany
}

\begin{abstract}
I review recent progress in simplicial quantum gravity in three and four dimensions, in particular new results on the phase structure of modified models of dynamical triangulations, the application of a strong-coupling expansion, and the benefits provided by including degenerate triangulations. In addition, I describe some recent numerical and analytical results on anisotropic crystalline membranes.
\end{abstract}

\section{Simplicial Quantum Gravity in D > 2}

The task of formulating a consistent theory of quantum gravity in four dimensions, hence unifying the two pillars of modern physics, general relativity and quantum mechanics, is a formidable one and still unresolved. Many different approaches have been tried (see e.g. Ref. [四), some have led to a deeper understanding of the problem but, as of yet, none can claim much success. In this article I will review the status, and recent progress, of one such approach, namely the attempt to make sense of an Euclidean path-integral quantization of general relativity via a discretization known as dynamical triangulations.

In Euclidean quantum gravity a path-integral is written as a formal sum over $D$-dimensional Euclidean geometries (metrics $g_{\mu \nu}$ )

$$
Z=\int \frac{\mathcal{D}[g]}{\operatorname{Vol}(\operatorname{diff})} \mathrm{e}^{-S_{E}\left[g_{\mu \nu}\right]}
$$

weighted by the Einstein-Hilbert action

$$
S_{E}\left[g_{\mu \nu}\right]=\frac{1}{16 \pi G} \int \mathrm{d}^{D} \xi \sqrt{|g|}(-R+2 \Lambda) .
$$

$G$ is the Newton's constant, $\Lambda$ the cosmological constant and $R$ the scalar curvature. However, this path-integral formulation faces some sever problems and unresolved questions:

- the theory is non-renormalizable,

- the action is unbounded from below, 
- adding higher-order curvature terms leads to renormalizable, albeit nonunitary, theory,

- how can Lorentzian signature be recovered from the Euclidean formulation.

A proposed interpretation is to view Eq. (2) as the first part of an effective action, valid in the infrared limit, and that fine-tuning of the parameters in the full effective theory might reveal a non-trivial ultraviolet stable fixed point where a consistent quantum theory of gravity might be defined [2] (a behavior akin to that of the non-linear $\sigma$-model). While it is true that non-trivial fixed points in four-dimensional field theories are rare, some interesting results obtained in $2+\epsilon$ dimensions actually lend support to this idea [3].

To address this proposal, a non-perturbative investigation of the theory is needed. One such is provided by a discretization of the path-integral Eq. (1), usually following a prescription due to Regge [4]. He proposed to approximate a smooth manifold with a given metric by a piecewise linear manifold (a triangulation), on which both parallel transport and the integral of curvature are well-defined. Choosing a sufficiently fine triangulation should provide a good approximation to any continuum manifold.

This idea has been applied to lattice gravity following one of two complimentary prescriptions, differing in how they implement the dynamical nature of the spacetime metric:

(a) Quantum Regge calculus

Use a piecewise linear manifold with fixed connectivity but with varying edge lengths [5].

(b) Dynamical Triangulations

Summing over triangulations with varying connectivity but fixed edge lengths [6].

In this review I will focus on the latter approach, for a recent review of quantum Regge calculus see e.g. Ref. [7].

\subsection{Dynamical triangulations}

In models of dynamical triangulations the continuum integral over diffeomorphism inequivalent metrics is replaced by a discrete sum over all possible decompositions (gluings) of equilateral $D$-simplexes along their $(D-1)$-dimensional faces into simplicial manifolds, while requering that the neighbourhood of each vertex is a $D$-ball (excluding pseudo-manifolds) [8, 9]. Fixing all edge lengths to a constant $a$ introduces a "reparametization invariant" cut-off in the model.

In this approach the Einstein-Hilbert action is approximated by a simple function of the numbers $N_{i}$ of $i$-dimensional simplexes on a given triangulations $T$. However, as these numbers are related through the Dehn-Sommerville and Eulers relations, the action can be taken to depend on only two of those numbers. The (grand-canonical) partition function becomes:

$$
Z(\mu, \kappa)=\sum_{N_{D}} \mathrm{e}^{-\mu N_{D}} \sum_{T \in \mathcal{T}_{N_{D}}} \frac{1}{C(T)} \mathrm{e}^{\kappa N_{D-2}},
$$



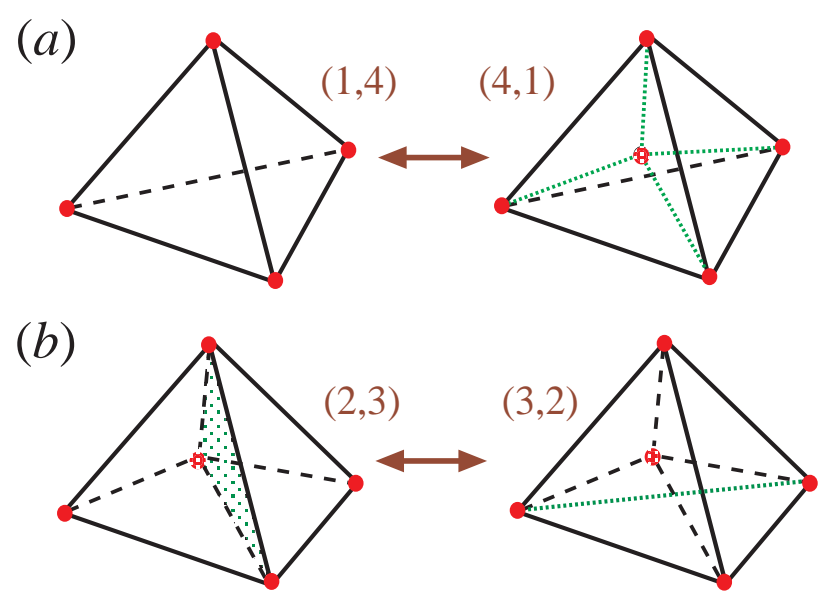

Figure 1: The $(p, q)$-moves in three dimensions: $(a)$ inserting/deleting a vertex, $(b)$ replacing a triangle by an edge and vice verse.

where $\mu$ and $\kappa$ represent the discrete cosmological and inverse Newtons's constants, and $C(T)$ is the order of the automorphishm group of $T$ - the number of equivalent labelings of the vertexes.

The second sum is over a suitable ensemble $\mathcal{T}$ of $D$-dimensional triangulations (of volume $N_{D}$ ). A priori, different ensembles can be used provided they lead to a well-defined partition function. While this leaves ample freedom in defining different ensembles, the topology of the triangulations must be fixed else the partition function is divergent in $N_{D}$. So far, most simulations in $D>2$ have used triangulations of spherical topology.

The main justification for the dynamical triangulation approach comes from two-dimensions where variety of exact solutions exist, complemented by extensive numerical results, and which agree with the solutions of continuum Liouville theory.I

\subsection{Simulation methods}

The partition function Eq. (3) is evaluated numerically using a Monte Carlo algorithm. The space of all triangulations is explored using a set of $D+1$ local (topology preserving) geometric moves - the $(p, q)$-moves [11]. In a $(p, q)$-move, where $p=D+1-q$, a $(q-1)$-subsimplex is replaced by its "dual" $(p-1)$-subsimplex, provided no manifold constraint is violated. The moves are ergodic in $D \leq 4$, i.e. any triangulation can be transformed into any other by an appropriate sequence of moves. An example of the $(p, q)$-moves in three dimensions is shown in $\mathrm{n} 1$.

In $D>2$ fluctuations in the volume $N_{D}$ are necessary for an ergodic updating procedure and it is customary to simulate the quasi-canonical partition function:

\footnotetext{
${ }^{1}$ In contrast, simulations of quantum Regge calculus in two dimensions fail to reproduce the known continuum results [10].
} 


$$
Z_{\bar{N}_{D}}(\mu, \kappa)=\sum_{N_{D}} \mathrm{e}^{-\mu N_{D}} \sum_{T \in \mathcal{T}_{N_{D}}} \frac{1}{C(T)} \mathrm{e}^{\kappa N_{D-2}-\delta\left(N_{D}-\bar{N}_{D}\right)^{2}}
$$

where the volume fluctuates around a target volume $\bar{N}_{D}$ and the quadratic potential term added to the action ensures that, for an appropriate choice of $\delta$, the fluctuations are small.

To explore the phase structure of model Eq. (4) powerful methods for probing the nature of the quantum geometry have been developed. The fractal structure of the triangulations is labeled by a set of critical exponents, e.g:

(i) The string susceptibility exponent $\gamma_{s}$ defines the singular behavior of the grandcanonical partition function: $Z(\mu) \approx Z_{\text {reg }}+\left(\mu-\mu_{c}\right)^{2-\gamma_{s}}$. This in turn implies that the canonical partition function behaves asymptotically as

$$
Z_{N_{D}}(\kappa) \sim \mathrm{e}^{\mu(\kappa) N_{D}} N_{D}^{\gamma_{s}(\kappa)-3}, \quad N_{D} \rightarrow \infty .
$$

A powerful method for measuring $\gamma_{s}$ in quasi-canonical simulations is provided by the size distribution of minbu's (minimal neck baby universes) [12] - a part of the triangulation connected to the rest via a minimal neck. By counting in how many ways a minbu of size $B$ can be connected to a surface of size $\left(N_{D}-B\right)$, the size distribution becomes

$$
\begin{aligned}
n_{N_{D}}(B) & \approx \frac{B Z_{B}\left(N_{D}-B\right) Z_{N_{D}-B}}{Z_{N_{D}}} \\
& \sim\left[\left(N_{D}-B\right) B\right]^{\gamma_{s}-2} .
\end{aligned}
$$

The distribution $n_{N_{D}}(B)$ is measured in simulations and $\gamma_{s}$ determined by a fit to Eq. (6).

(ii) The fractal or Hausdorff dimension $d_{H}$ measures the intrinsic "dimensionality" of the triangulations and is defined by the volume of a geodesic ball with radius $r$ : $v(r) \sim r^{d_{H}}$. The geodesic distance is defined as the shortest path $d_{i j}$, between two vertexes, traversed along links, either on the triangulations or its dual graph.

To measure the fractal dimension $d_{H}$ one explores the scaling behavior of the vertex-vertex, or two-point, correlation function,

$$
g_{N_{D}}(r)=\frac{1}{N_{D}}\left\langle\sum_{i, j} \delta\left(d_{i j}-r\right)\right\rangle_{T}
$$

which counts the number of vertexes at distance $r$ from a marked vertex $i$. Assuming that the only relevant length-scale in the model is defined by $N_{D}^{1 / d_{H}}$, general scaling arguments 13] imply that

$$
g_{N_{D}}(r) \sim N_{D}^{1-1 / d_{H}} F\left(\frac{r}{N_{D}^{1 / d_{H}}}\right) .
$$

The optimal scaling of distributions $g_{N_{D}}(r)$, corresponding to different volumes, onto a single scaling curve defines $d_{H}$. 


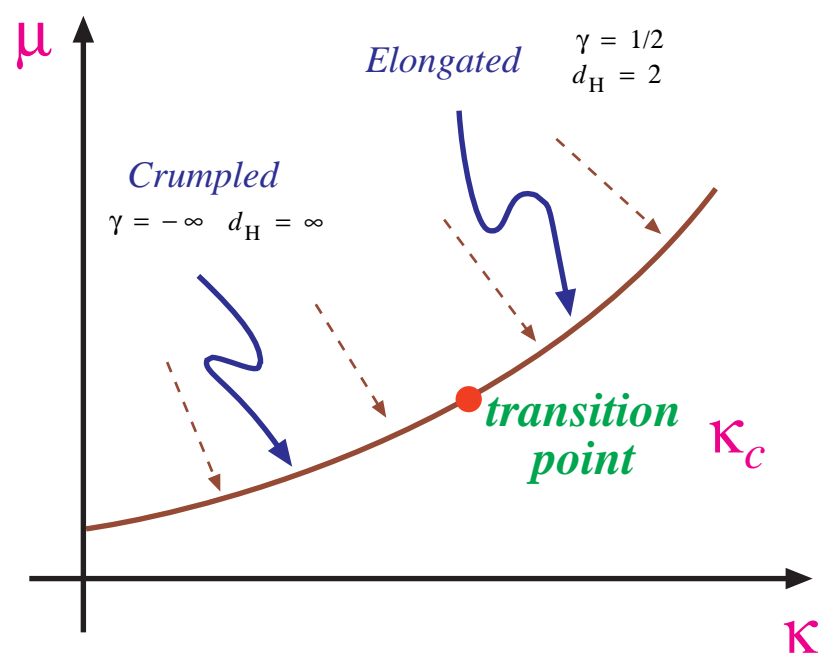

Figure 2: A schematic phase diagram of pure simplicial gravity in $D>2$.

\subsection{The pure gravity phase structure}

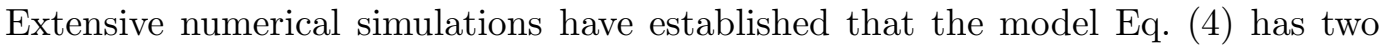
distinct phases, both in three and four dimensions. There is a strong-coupling $($ small $\kappa)$ crumpled phase and a weak-coupling (large $\kappa$ ) elongated phase. The two phases are separated by a phase transition at critical value of the inverse Newton's constant $\kappa_{c}$.

For $\kappa<\kappa_{c}$ the internal structure collapses and the crumpled phase is characterized by a singular structure, i.e. a small set of sub-simplexes connected to an extensive fraction of the total volume [14]. In $D=4$ this structure consists of two singular vertexes, with local volumes $\omega_{v}^{\prime}$ that grows linearly with the volume $N_{4}$, joined by a sub-singular edge; $\omega_{e}^{\prime} \sim N_{4}^{2 / 3}$. In $D=3$ there is only a sub-singular vertex with $\omega_{v}^{\prime} \sim N_{3}^{1 / 3}$. The geodesic distance between any two vertexes always stays at the level of the lattice cut-off. This prevents any sensible continuum limit in this phase - the quantum geometry is essentially zero-dimensional. In addition, large geometric out-growths are suppressed and, at least formally $2, \gamma_{s}=-\infty$.

In the elongated phase the internal geometry is composed of bubbles glued together via small necks into tree-like structures — branched polymers — with $d_{H}=2$ and $\gamma_{s}=1 / 2$. The neck size does not grow with the volume and the internal geometry is essentially one-dimensional.

As the fractal structures described above are somewhat pathological, it is unlikely that an interesting continuum limit exist in either of the two phases. The initial hope was, however, that the transition point $\kappa_{c}$ would correspond to a non-trivial fixed point where a sensible continuum limit could be defined. However, the transition turned out to be discontinuous, both in three [15, 16] and four [17] dimensions; this is demonstrated in Figure 3.

\footnotetext{
${ }^{2}$ In the crumpled phase the sub-leading asymptotic behavior is $Z_{N_{D}}(\mu) \sim \exp \left(\mu N_{D}+a N_{D}^{b}\right)$, $b<1$, rather than Eq. (5). Hence $\gamma_{s}$ is not well defined.
} 

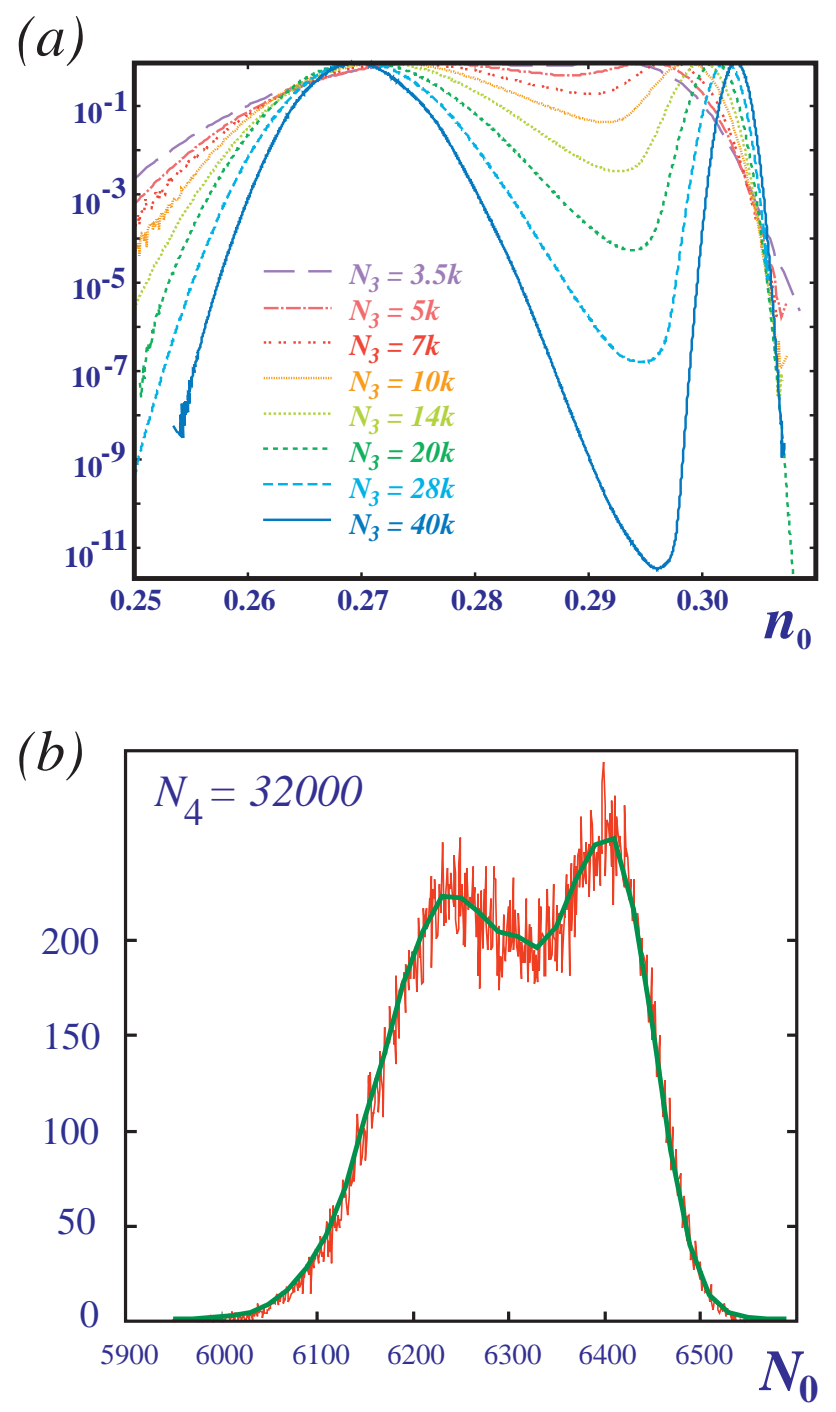

Figure 3: Histogram of the energy density, $n_{0}=N_{0} / N_{D}$, for pure simplicial gravity at the transition $\kappa_{c}$. Both in (a) three [16], and (b) four [17] dimensions a two state signal is observed, characteristic of a discontinuous phase transition.

In four dimensions the transition is weakly first order and, consequently, hard to observe - it is only manifest on large volumes, $N_{4} \gtrsim 32.000$. As present day computers limit practical simulations to $N_{4} \lesssim 10^{5}$, this poses a serious problem for any numerical investigation of the model Eq. (3). 


\section{Modified Models of Simplical Gravity}

The discontinuous nature of the phase transition implies that the model Eq. (3) does not posses a continuum limit where a theory of gravity could be defined. Does this mean that there is something inherently wrong with the dynamical triangulation approach, or is the discretization Eq. (3) simply too naive - some fine-tuning of additional parameters might be needed. The success of two-dimensional models of dynamical triangulations, where the critical behavior is remarkable insensitive to details of the discretization, might be misleading. The theory of gravity in higher dimensions is more complicated and more carefully defined discretization may be necessary.

This has led to investigations of modified models of simplicial gravity; modified either by changing the relative weight of the triangulations with e.g. a measure term, or by coupling matter fields to the theory. Although preliminary investigations of such modified models did not observe any qualitative change in the critical behavior [18, 19], more recent numerical simulations 20, 16, 21] have revealed that such modifications do indeed influence the phase structure: The phase transition appears to soften, or even disappear. Moreover, a new crinkled phase is observed, characterized by internal geometry closer to what one might expect of smooth manifolds.

Two such modified models of simplicial gravity have recently been investigated in $D>2$ :

(a) A modified measure:

$$
Z_{N_{D}}(\kappa, \beta)=\sum_{T \in \mathcal{T}_{N_{D}}} \prod_{i \in N_{D-2}} o\left(n_{i}\right)^{\beta} \frac{\mathrm{e}^{\kappa N_{D-2}}}{C(T)},
$$

where $o\left(n_{i}\right)$ is the local volume of an sub-simples $i$ - the number of $D$-simplexes containing $i$.

(b) Coupling to $f$ copies of matter fields:

$$
Z_{N_{D}}(\kappa, f)=\sum_{T \in \mathcal{T}_{N_{D}}} \frac{\mathrm{e}^{\kappa N_{D-2}}}{C(T)}\left(Z_{\mathrm{M}}(T)\right)^{f}
$$

where $Z_{M}$ is the matter partition function.

In Ref. [21] the particular case of vector fields coupled to four-dimensional simplicial gravity was investigated:

$$
\begin{aligned}
Z_{\mathrm{M}}(T) & =\int \prod_{l \in T} \mathrm{~d} A(l) \mathrm{e}^{-S_{\mathrm{VF}}} \\
S_{\mathrm{VF}} & =\sum_{t_{a b c}} o\left(t_{a b c}\right)\left[A\left(l_{a b}\right)+A\left(l_{b c}\right)+A\left(l_{c a}\right)\right]^{2}
\end{aligned}
$$

where $A(l)$ are non-compact $U(1)$ gauge fields residing on the edges $l, A\left(l_{a b}\right)=-A\left(l_{b a}\right)$, and the sum is over all triangles $t_{a b c}$ in the four-dimensional triangulation. 


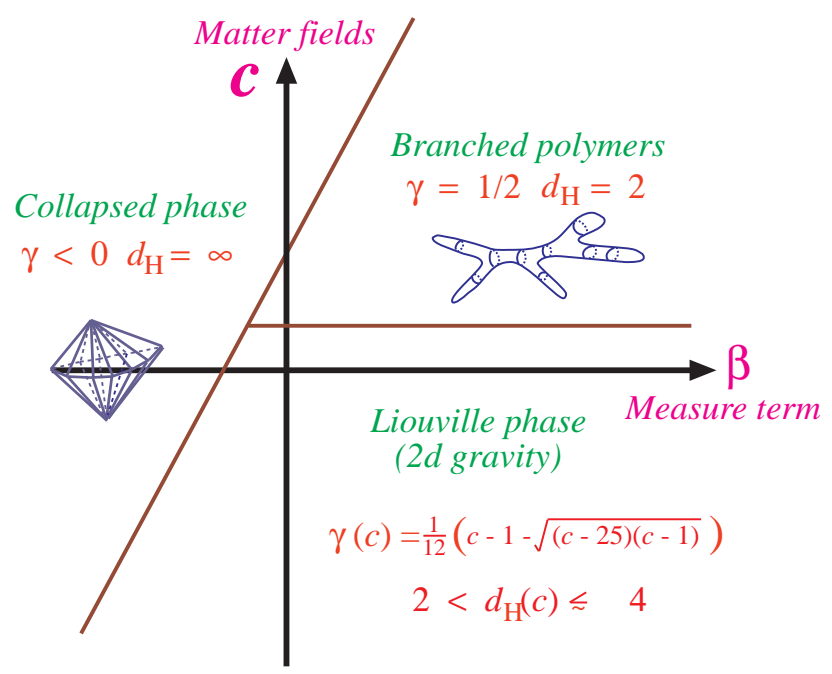

Figure 4: The phase structure of two-dimensional simplicial gravity coupled to matter fields and/or a measure term.

\subsection{Lessons from two dimensions}

Before discussing the phase structure of the models Eqs. (9) and (10) for $D>2$, it is worth considering the impact the two modifications have on simplicial gravity in two dimensions. [?

In two dimensions modifying the measure has no effect on the critical behavior, except for large negative values of the coupling $\beta$ where formations of large curvature defects is enhanced and there is a cross-over to a crumpled phase, dominated by intrinsically collapsed configurations.

In contrast, adding matter fields changes the critical behavior. For matter fields with central charge $c \leq 1$ this corresponds to coupling conformally invariant matter to $2 D$-gravity (Liouville theory). In this phase the fractal structure displays a remarkable degree of universality; it only depends on the total central charge of the matter fields: $-\infty \leq \gamma_{s}(c) \leq 0$, and $2 \leq d_{H}(c) \lesssim 4$. For $c>1$, on the other hand, the fractal structure degenerates into branched polymers. This is understood qualitatively as a condensation of spiky configurations [22]; the free energy of spikes, $F_{\text {spike }} \sim(1-c) \log (1 / a)$, estimated from the dynamics of the conformal factor, shows that such singular configurations dominate the partition function for $c>1$.

What does this imply for the phase diagram in higher dimensions? Is it possible that the similarity between the phase structure for pure gravity in $D>2$ and the $c>1$ region in Figure 4 is not coincidental. That a suitable tuning of the parameters $\beta$ or $f$, could lead to some higher-dimensional analog of the Liouville phase?

This scenario is supported by recent analysis in Ref. 23] which suggest that something analogous to the " $c=1$ barrier" might exist in four dimensions. Using

\footnotetext{
${ }^{3}$ In $D=2$ the phase diagram simplifies as the Einstein term in the action is a topological invariant and the dynamics is insensitive to the corresponding coupling constant (as long as the topology of the manifold is fixed).
} 


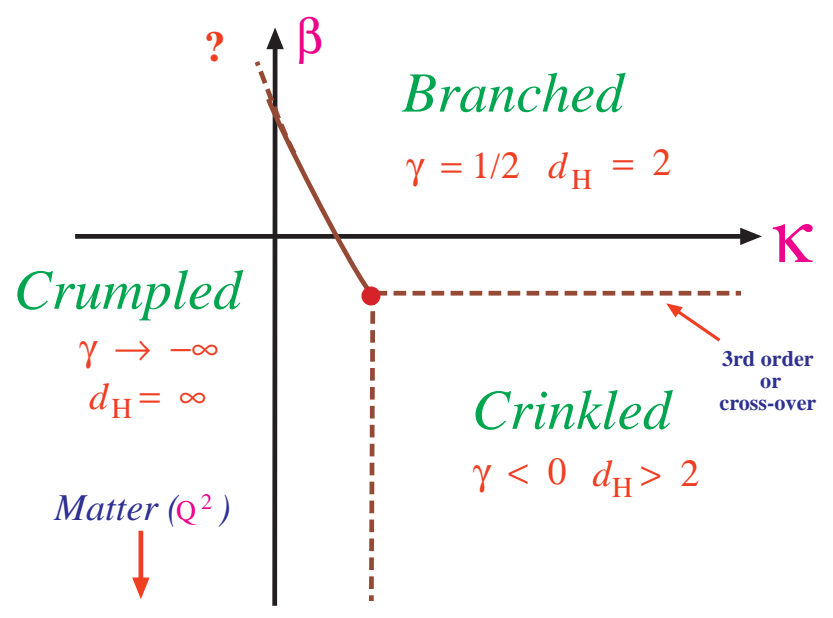

Figure 5: A schematic phase diagram of modified models of simplicial gravity for $D>2$.

an effective action for the conformal factor, they calculate the free energy of spikes:

$$
F_{\text {spike }} \sim \frac{1}{2}\left(Q^{2}-8\right) \log \left(\frac{1}{a}\right) .
$$

$Q^{2}=\frac{1}{180}\left(N_{S}+\frac{11}{2} N_{F}+62 N_{V}-28\right)+Q_{\text {Grav }}^{2}$ plays the role of a central charge and $N_{S, F, V}$ refers to the number of massless scalar, fermion and vector fields contributing to the trace anomaly. A one-loop calculation of the contribution from the transverse gravitons, $Q_{\text {Grav }}^{2} \approx 7.9$, suggests that in the absence of matter fields the theory has no sensible stable vacuum. It is intriguing, however, that the contribution of matter and ghost fields appears in $D=4$ opposite to that found in $D=2$; in four dimensions adding matter fields might actually stabilize the theory!

\subsection{Modified phase structure in $\mathrm{D}>2$}

Motivated by these considerations, extensive numerical investigations have been carried out on the models Eqs. (9) and (12) [16, 20, 21]. In addition to MC simulations, a method for calculating the strong-coupling expansion of simplicial gravity in $D>2$ has been developed [21], and has proved invaluable for a qualitative view of the impact different modifications have on the critical behavior. The results of these investigations, which suggest a phase diagram akin to Figure 5, can be summarized as follows:

(i) As several vector fields are coupled to simplicial gravity (or the curvature fluctuation are enhanced by modifying the measure) the discontinuous phase transition (solid line), separating the crumpled and the elongated phases, is softened and eventually, at some value $f \gtrsim 3(\beta \lesssim-1)$, either becomes continuous or disappears altogether.

(ii) At the same value of $f(\beta)$ the polymerizations of the geometry is suppressed, the elongated phase disappears, and a new crinkled phase appears. The crinkled phase is separated from the crumpled and elongated phases by lines of either continuous phase transitions or by a crossover region (dashed lines). 


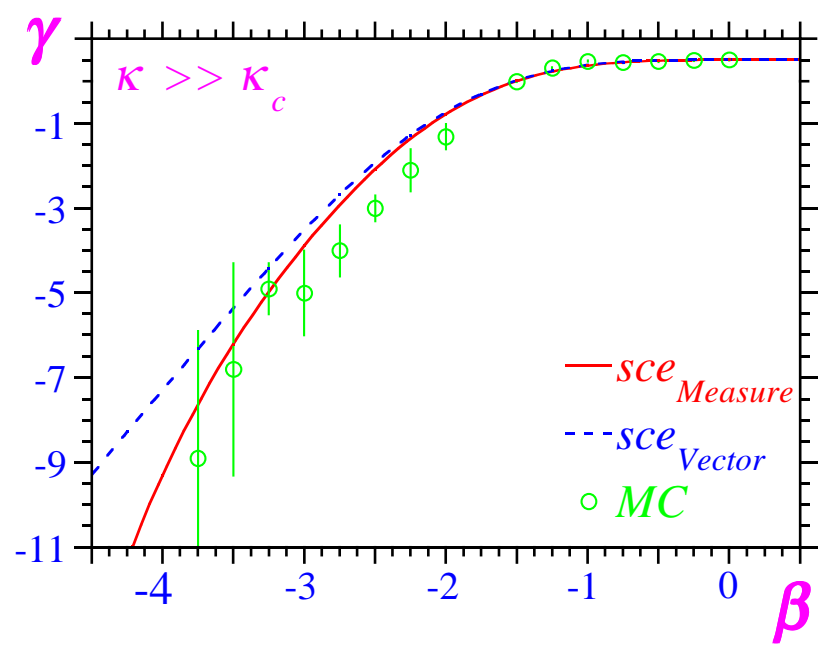

Figure 6: The exponent $\gamma_{s}$ versus a modified measure (solid line) or coupling to matter fields (dashed) from the strong-coupling expansion for $\kappa \gg \kappa_{c}$. The circles are from MC simulations of the model Eq. (9) for $\kappa=4.5$ [21].

In contrast to two dimensions, a remarkable degree of universality appears in simplicial gravity in $D>2$; all "reasonable" modifications seem to lead to the same qualitative phase structure Figure 5 [21].

The nature of the crinkled phase has been explored for $D=4$, both in MC simulations and from the strong-coupling expansion [21]. As the number of vector fields $f$ is increased (or $\beta$ decreased), the string susceptibility exponent $\gamma_{s}$ decreases (Figure 6). Moreover, modulo some trivial re-scaling: $\beta=-\frac{1}{2} f-\frac{1}{4}$, the results for the two models are identical. Measurements of the fractal dimension in the crinkled phase yield $d_{H} \approx 4$.

At the transition lines separating the crinkled phase from the rest of the phase diagram (the dashed lines), no divergence is observed in the specific heat, only a cusp. It is thus difficult to determine in MC simulations if these are soft continuous transitions (third or higher order) or if this represents a smooth cross-over to different a fractal structure. However, a soft continuous phase transition would not be unexpected; coupling to geometric disorder tends to soften phases transitions. For example, most transitions for matter coupled to $2 D$-gravity are of third order.

How should one interpret the phase structure Figure 5? Does the crinkled phase play a role analogous to the Liouville phase in two dimensions, or is the internal geometry just as pathological in this phase as in the crumpled and elongated phases? Alternatively, is a continuum limit possible at some of the observed transition lines; provided, of course, they correspond continuous phase transitions? Further investigations will hopefully shed some light on these issues. 


\subsection{The strong-coupling expansion}

The strong-coupling expansion - an explicit enumeration of all triangulations up to a given volume - provides a powerful tool for exploring the phase structure of different modifications of simplicial gravity. It has previously been used in two dimensions, where a matrix-model formulation allows a recursive construction of all triangulations [24]. Combined with an appropriate series extrapolation method, the strong coupling expansion yields, for some models, very accurate estimates of the critical exponents.

In $D>2$ a recursive algorithm for generating all triangulations is not available. Instead an alternative procedure constructing the series in numerical simulations has been developed [21, 25]:

(i) Identify all distinct triangulations $T$ using $\mathrm{MC}$ simulations and a sufficiently complicated "hash" function.

(ii) Calculate the corresponding symmetry factor $C(T)$ by comparing all permutations of the vertex labels.

(iii) For each triangulation $T$ calculate the appropriate contribution from matter fields and/or a modified measure.

The hash function $f(T)$ plays a vital role in the identification. It should be sufficiently complicated to distinguish between combinatorially inequivalent triangulations, yet simple enough to be repeatedly calculated. The identification is verified by comparing the calculated symmetry factors $C(T)$ to the relative frequency with which different triangulation appear in the MC simulations. In practice, the identification procedure is much simplified by observing that only triangulations not reducible by any of the volume decreasing geometric moves have to be considered in the MC; all others are systematically constructed from smaller ones. In practice $99.9 \%$ of the triangulations turn out to be reducible.

This method has been used to calculate the first 15 to 20 terms in the strongcoupling expansion of the partition function Eq. (3), both in three and four dimensions. This corresponds to roughly $10^{6}$ distinct triangulations. Given the triangulations it is easy to calculate the contribution from different types of matter fields, or from a modified measure, to the partition function. To determine the critical behavior, appropriate series extrapolation methods are used, e.g. the ratio method [24], in which higher order corrections to the partition function are systematically eliminated.

The method is, however, limited to the region of the phase-space where the partition function is rapidly convergent. In cases where the sub-leading corrections to the partition function are not analytic, such as in the presence of a confluent singularity, the extrapolation fails to converge. In simplicial gravity the strong-coupling expansion has provided valuable informations about the nature of the elongated and crinkled phases; however, it has been of limited use in the crumpled phase and in the transitions region. 


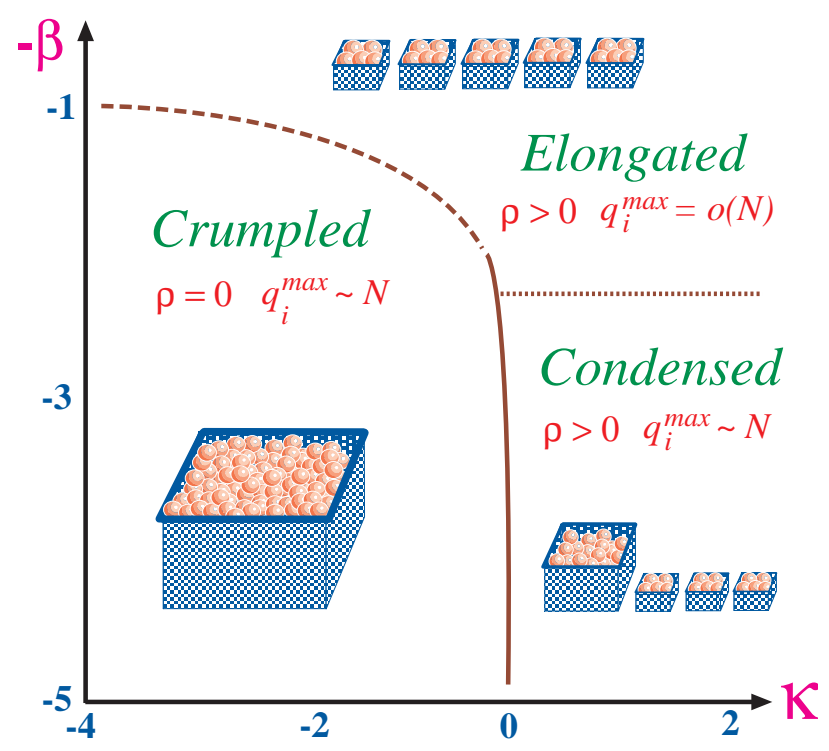

Figure 7: The phase diagram of balls-in-boxes.

\section{4 "Balls in boxes"}

Many of the characteristics of the phase structure of simplicial gravity, described in the previous sections, are captured by a simple mean-field model - the balls-inboxes model [26]. This model consists of fixed number $N$ of balls distributed into a variable number $M$ of boxes, $1 \leq M \leq M_{\max }$. In its partition function,

$$
Z_{N}(\kappa, \beta)=\sum_{m=1}^{M_{\max }} \mathrm{e}^{\kappa m} \sum_{q_{1}, \ldots, q_{m}} p\left(q_{1}\right) \ldots p\left(q_{m}\right) \delta_{q_{1}+\ldots+q_{m}, N},
$$

the partitions of balls are weighted by the product of one-box weights $p\left(q_{i}\right)=q_{i}^{-\beta}$, where $q_{i}=1,2, \ldots$ is the number of balls in box $i$. This particular choice of weights mimics the modified measure, Eq. (9), introduced in simplicial gravity.

The model Eq. (13) derives its non-trivial features from the constraint on the total number of balls. Introducing the average density of balls $\rho=M / N$, the "curvature", there is phase transition at a critical density $\rho_{c}$. This transition separates an elongated (fluid) phase, with the balls evenly distribution among the boxes, from a crumpled phase, where the balls "condensate" into one singular box (akin to BoseEinstein condensation). The transition is first order for $\beta \in(2, \infty)$, whereas it is continuous for $\beta \in(1,2]$.

By introducing an additional kinematic bound on the average density of balls per box, $\rho<\rho_{\text {art }}$, a new condensed phase appears in the model for $\beta \gtrsim 2.5$ [27]. This is shown in Figure 7 . In this phase, which resembles the crinkled phase of simplical gravity, the model is neither crumpled nor fluid. The average density per box is finite, $\langle r\rangle>0$; however, there is a singular box in the system which captures a finite number of the balls: $\left\langle q_{\text {sing }}\right\rangle / N>0$. 
It is remarkable how such a simple model successfully describes many of the geometric features of simplicial gravity in $D>2$, e.g. the appearance of a singular vertex and the phase structure, while ignoring all the non-trivial information encoded in the connectivity of the triangulations.

\section{Degenerate Triangulations}

In the partition function Eq. (3), $\mathcal{T}$ denotes a suitable ensemble of triangulations included in the sum. Different ensembles are defined by imposing various restriction on how the simplexes are glued together. Provided this leads to a well-defined partition function, and as long as this difference is only at the level of the discretization, one expects different choices of $\mathcal{T}$ to lead to the same continuum theory in the thermodynamic limit. This is, of course, not true if the partition function is divergent as is the case even in two dimensions if the topology is not restricted.

Traditionally, simulations of simplicial gravity in $D>2$ have used an ensemble of combinatorial triangulations $\mathcal{T}_{C}$. In a combinatorial triangulation every $D$-simplex is uniquely defined by a set of $D+1$ distinct vertexes - it is combinatorially unique. This implies that two distinct simplexes have at most one face in common; this amounts to exclude both tadpoles and self-energy diagrams in the corresponding dual graph. Easing this restriction, allowing either multiple connected simplexes or simplexes glued onto themselves, defines ensembles of restricted and maximally degenerate triangulations, $\mathcal{T}_{D_{R}}$ and $\mathcal{T}_{D_{M}}$, respectively [30]. In the former ensemble to each simplex there is associated a set of $D+1$ distinct vertexes; however, the same set of vertexes may be shared by any number of distinct simplexes. In contrast, in a maximally degenerate triangulation even the set of vertexes associated to a simplex may be degenerate, i.e. it may contain the same vertex more than once. Clearly, $\mathcal{T}_{C} \subset \mathcal{T}_{D_{R}} \subset \mathcal{T}_{D_{M}}$. Figure 8 shows two-dimensional examples of those three different types of triangulations.

In two dimensions the model Eq. (3) defined with those different ensembles is solvable as matrix model and, in all three cases, it yields the same continuum theory [28]. Then why consider different ensembles; why not use the one most convenient in the simulations? It turns out that the finite-size effects depend strongly on the ensemble used [29]. In particular, the finite-size effects are considerable reduced the less restrictions are placed on the triangulations. An example of this is the volume dependence of the effective magnetization exponent, $\beta / \nu d_{H}$, for an Ising model coupled to $2 D$-gravity (Figure 9 ). This reduction in the finite-size effects is understandable - with a large ensemble of triangulations it is easier to approximate a given continuum geometry using triangulations of finite volume.

\section{1 $\mathrm{D}>2$}

It is only recently that simulations of simplicial gravity in higher dimensions have been extended to include degenerate triangulations. In three dimensions both restricted and maximally degenerate triangulations have been investigated [30]; however, only the former ensemble was found to lead to a well-defined partition function 

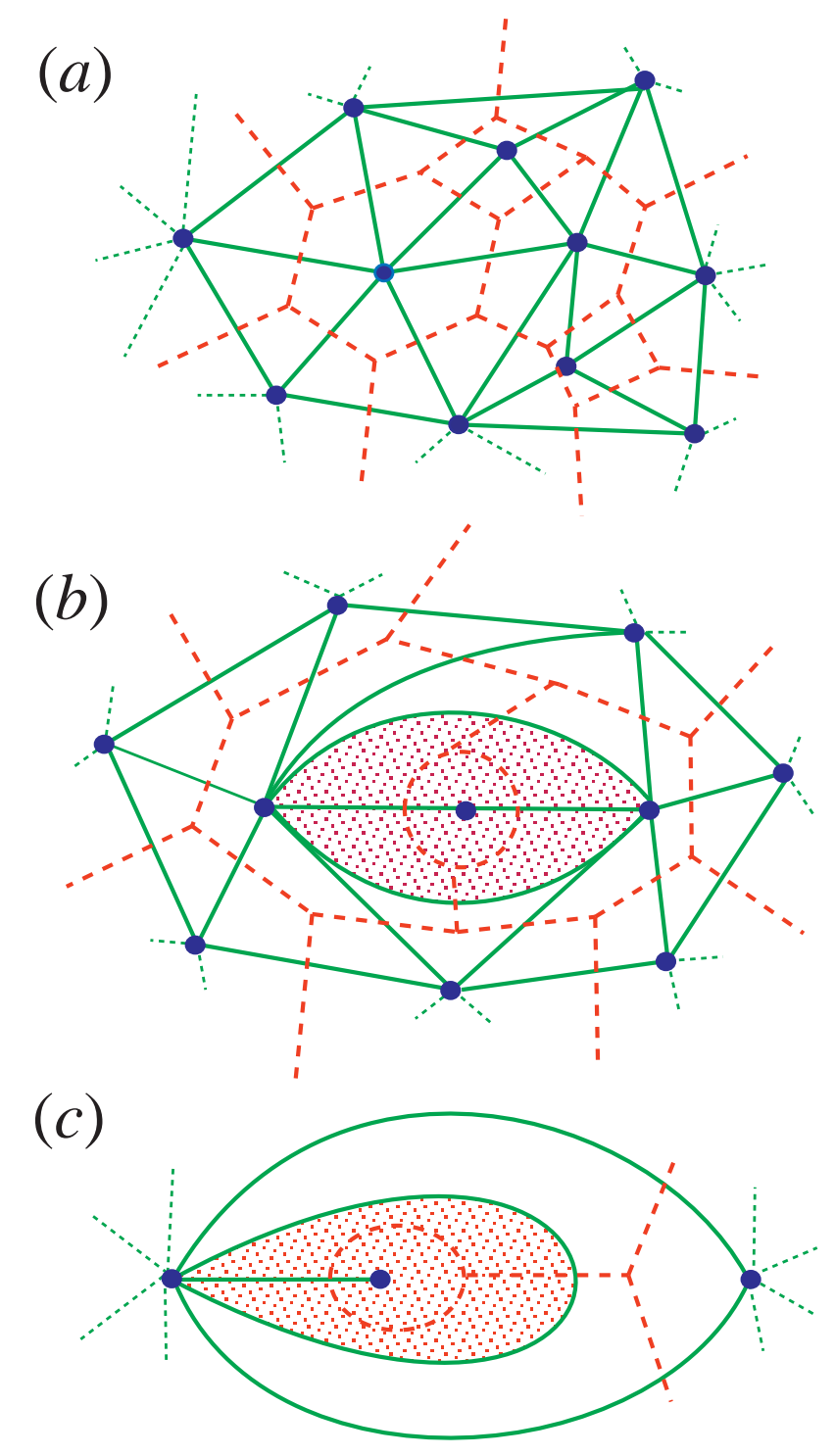

Figure 8: Three types of two-dimensional triangulations: $(a)$ combinatorial, $(b)$ restricted degenerate, and $(c)$ maximally degenerate. The dashed lines indicate the corresponding dual graphs. 


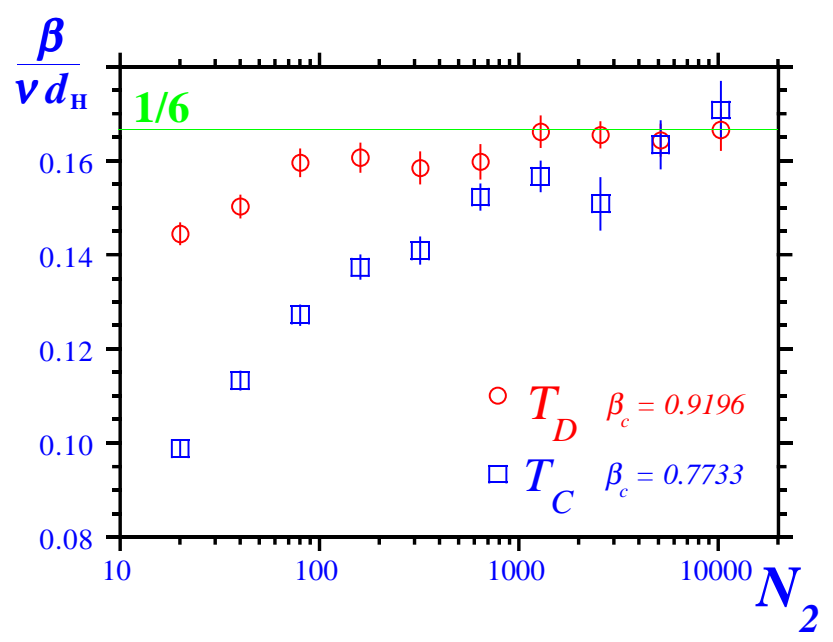

Figure 9: The effective magnetization exponent, $\beta / \nu d_{H}$, for an Ising model coupled to $2 D$-gravity versus volume [29], both for combinatorial (squares) and degenerate (circles) triangulations 29]. The theoretic value is $1 / 6$.

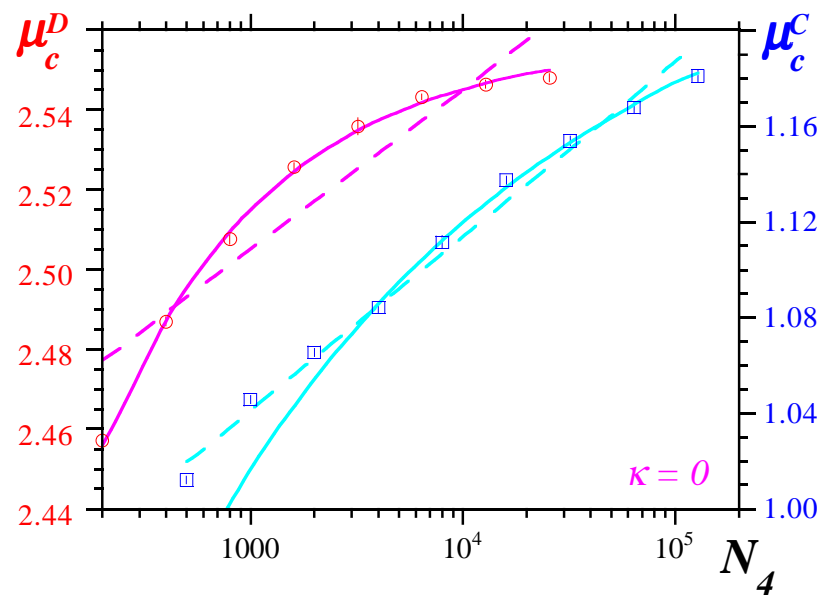

Figure 10: The pseudo-critical cosmological constant $\mu_{c}\left(N_{4}\right)$ for ensembles of $4 D$ combinatorial (squares) and degenerate (circles) triangulations. Included are fits assuming an power-law convergence (solid lines) and a logarithmic divergence (dashed lines) 31. 
Eq. (3). More recently restricted degenerate triangulations have also been used in four dimensions [31].

Qualitatively, the phase structure does not change when degenerate triangulations are included in the model Eq. (3). There is still a small $\kappa$ crumpled phase and a large $\kappa$ elongated phase, separated by a discontinuous transition. However, just as in two dimensions the finite-size effects appear much reduced, especially in the crumpled phase. An example of this is the pseudo-critical cosmological constant $\mu_{c}\left(N_{D}\right)$, which converges much more rapidly for the ensemble $\mathcal{T}_{D_{R}}$ than for $\mathcal{T}_{C}$. This is shown in Figure 10 for $D=4$ and $\kappa=0$.

This important observation demonstrates (numerically) an exponential bound on the number of degenerate $4 D$ triangulations of fixed volumf - a necessary condition for the convergence of the partition function Eq. (3). This is demonstrated in Figure 10 by two fits: one fit assumes an exponential bound on the canonical partition function (solid), the other a super-exponential growth (dashed). Whereas for combinatorial triangulations the fits are somewhat inconclusive, for degenerate triangulations a super-exponential growth is definitely ruled out [31. And, as $\mathcal{T}_{C} \subset \mathcal{T}_{D}$, this automatically implies a bound on the entropy of combinatorial triangulations as well.

\section{Physical Membranes}

The study of the elastic and geometrical properties of physical membranes, twodimensional surfaces with an extrinsic curvature embedded in $\mathbf{R}^{3}$, is a rapidly advancing field with a nice interplay between experiments, analytical theory and computer simulations (see e.g. Refs. 33, 34]). Viewed as two-dimensional generalization of one-dimensional chains, membranes show a rich behavior on mesoscopic length scales. They can be classified according to their internal structure:

(a) Polymerized or crystalline membranes have fixed internal structure and are characterized by both long-range orientational and translational order.

(b) Fluid membranes, with dynamical internal connectivity and no internal order.

An example of the former is the spectrin cytoskeleton of red bloods cells, whereas bilayers of amphiphiles such as phospholipids generally exhibit liquid-like behavior. Physical membranes encountered in nature are, of course, self-avoiding; a property pivotal to many aspects of their phase structure. They are, however, commonly studied as self-intersecting (phantom) membranes, both for simplicity and as statistical system interesting in their own right.

It is by now well established, both by analytical and computational work, that crystalline membranes exhibit a low-temperature flat (ordered) phase and, at least for phantom membranes, a high-temperature crumpled phase [35]. The existence of an ordered phase in a two-dimensional system with a continuous symmetry and an (apparent) local interaction is remarkable given the Mermin-Wagner theorem.

\footnotetext{
${ }^{4}$ The existence of an exponential bound has been somewhat controversial for the ensemble of combinatorial triangulations in four dimensions [32].
} 


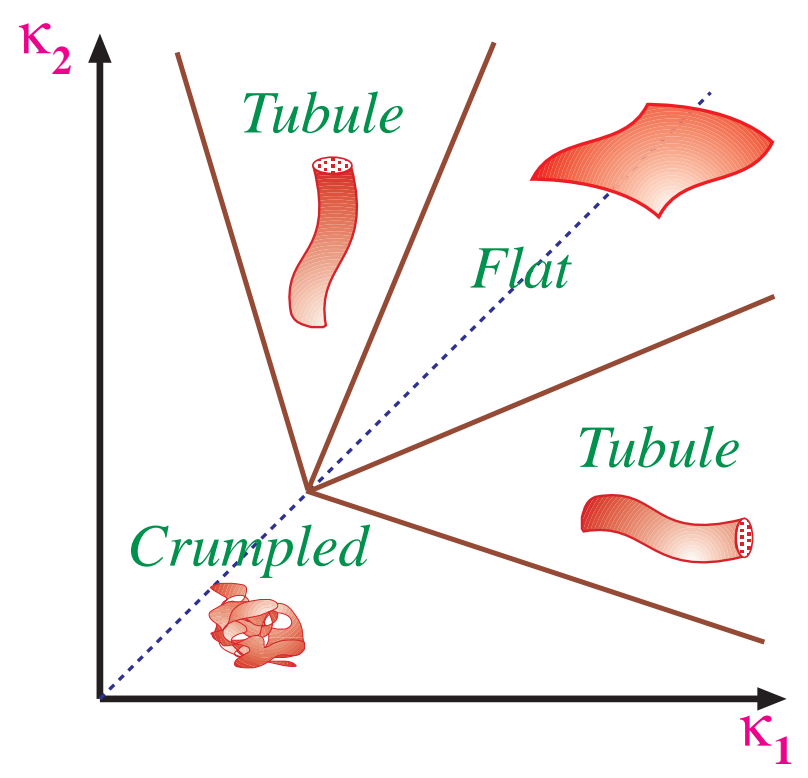

Figure 11: A schematic phase diagram of a phantom anisotropic crystalline membrane.

What stabilizes the flat phase in a crystalline membrane are the out-of-plane fluctuations that couple to the in-plane "phonon" degrees of freedom - bending of the membrane is accompanied by internal stretching.

The same argument for a stable flat phase does not apply in the case of a fluid membrane where the internal stretching can be compensated by a flow of "particles" into the distorted area, thereby "screening" the curvature fluctuations from the rest of the membrane. The absence of a stable flat phase is supported by renormalization group analysis of models of fluid membranes, which suggest that at large distances the bending rigidity becomes irrelevant [36]. This is, however, contradicted by numerous numerical simulations of fluid membranes that indicate a crumpling transition, akin to that of its crystalline counterpart [37].

\subsection{Including anisotropy}

The above discussion applies to isotropic membranes. Remarkable, adding intrinsic anisotropy to a model of crystalline membrane profoundly influences the global phase diagram [38]. Most notably, a new tubular phase appears, characterized by the presence of long-range orientational order in one direction only - in the transverse directions the membrane is crumpled. For a phantom membrane, the tubular phase separates the existing crumpled and flat phases; only an isotropic membrane passes directly from a flat to a crumpled state (Figure 11). Analogous to a flat membrane, a tubular membrane is stabilized by the transverse stretching energy cost of bending fluctuations in the extended direction.

The existence of a tubular phase has been confirmed in recent numerical simulations on a crystalline membranes [39]. The membrane is discretized on a triangular

\footnotetext{
${ }^{5}$ Simulations of physical membranes are notoriously difficult due to very long auto-correlation
} 


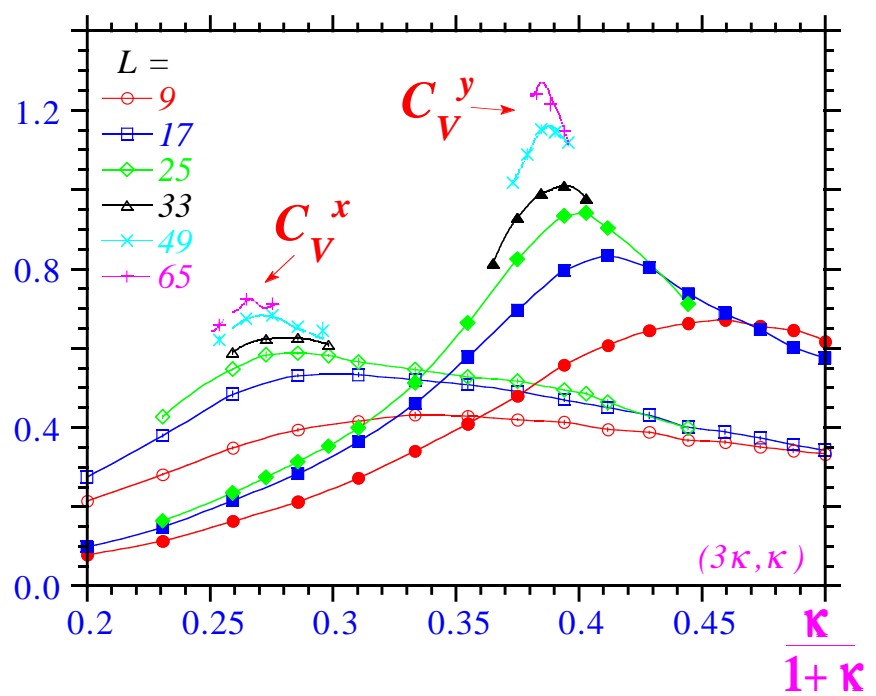

Figure 12: The fluctuations in the two bending energy terms: $C_{V}^{x}$ and $C_{V}^{y}$, for a phantom anisotropic crystalline membrane, versus $\left(\kappa_{1}, \kappa_{2}\right)=(3 \kappa, \kappa)$ [39].

mesh, with the topology of a disk, and the interactions modeled by Hamiltonian composed of two terms: a Gaussian pair potential between neighboring nodes, and a bending energy introduced as ferromagnetic interaction between normals $\mathbf{n}_{a}$ on adjacent triangles:

$$
\mathcal{H}[\mathbf{r}]=\sum_{\left\langle\sigma \sigma^{\prime}\right\rangle}\left|\mathbf{r}_{\sigma}-\mathbf{r}_{\sigma^{\prime}}\right|^{2}-\kappa_{1} \sum_{\langle a b\rangle}^{(x)} \mathbf{n}_{a} \cdot \mathbf{n}_{b}-\kappa_{2} \sum_{\langle a b\rangle}^{(y)} \mathbf{n}_{a} \cdot \mathbf{n}_{b} .
$$

The anisotropy is introduced as different bending rigidity in the two intrinsic directions, $x$ and $y$.

The phase diagram of the model Eq. (14) was explored, for different bending rigidities $\left(\kappa_{1}, \kappa_{2}\right)$, and evidence for two distinct phase transition - a crumpled-totubular and tubular-to-flat - is observed in the fluctuations in the two bending energy terms: $C_{V}^{x}(\kappa)$ and $C_{V}^{y}(\kappa)$ (Figure 12).

In the tubular phase, the tubule cross-section radius $R_{\perp}^{G}$ (the radius of gyration) and the undulations $h_{\text {rms }}$ transverse to its average axis of orientation, scale like: $R_{\perp}^{G} \sim L^{\nu_{F}}$ and $h_{\mathrm{rms}} \sim L^{\zeta}$, where $\nu_{F}$ and $\zeta$ are the size (Flory) and roughness exponents. MC simulations of a tubular membrane give: $\nu_{F}=0.269(7)$ and $\zeta=$ 0.850(40) [39], compared to the theoretical predictions: $\nu_{F}=\frac{1}{4}$ and $\zeta=1$ 38]. Notice that $\zeta=1$ implies that a phantom tubule is only marginally stable with respect to the fluctuations in the transverse directions; the tubule almost crumples.

times present in standard MC simulations. These auto-correlations are, though, much reduced by applying more powerful updating methods such as overrelaxation or unigrid algorithms in the simulations 40 . 


\subsection{Self-avoiding membranes}

The more physical self-avoiding membranes present more of a challenge, especially for numerical simulations, due to the non-local nature of the self-avoiding constraint. For a flat isotropic crystalline membrane self-avoidance is expected to be irrelevant; self-avoidance is, however, expected to suppress the crumpled phase. Hence only flat self-avoiding isotropic membranes are expected to exist; this is supported by numerical simulations.

For membranes crumpled in one direction only, self-avoidance is less constraining and the tubular phase is expected to survive in the more physical self-avoiding case [38, 41, 42]. However, unlike for a flat membrane self-avoidance is expected to be relevant for tubules. Adding a non-vanishing self-avoiding coupling leads to a flow in the couplings to a new critical (infrared stable) fixed point, distinct from the one that governs the critical behavior of a phantom tubule. This fixed point structure is supported by renormalization group analysis [38, 42]. An improved one-loop calculations of the critical exponents $\nu$ and $\zeta$, in an $\epsilon$-expansion around the upper critical dimension $d_{c}^{u p}=11$, gives: $\nu=0.62$ and $\zeta=0.8$ [42].

It is of great interest, albeit very challenging, to verify this critical behavior in numerical simulations of a self-avoiding anisotropic membrane. This work is underway [43]. Preliminary results indicate the existence of only one phase transition, from a tubular to a flat phase; as expected a crumpled phase is not observed.

\section{Outlook}

(i) Much work is needed before the phase structure of modified models of simplicial gravity in $D>2$ is fully understood. The nature of the crinkled phase, and of the observed phase transitions, must be established before any statements can be made about the relevance the observed phase structure Figure 5 has for any potential continuum theory of quantum gravity.

(ii) Degenerate triangulations might prove a valuable tool in any such investigations. In addition to reducing the finite-size effects in simulations, they also serve as a consistency check on any observed phase structure in simplicial gravity - any critical behavior that is relevant in a continuum limit should be independent of the ensemble of triangulations used in defining the model.

(iii) Anisotropic crystalline membrane have a surprisingly rich phase structure, some of which has been revealed through analytic calculations and/or numerical simulations. To extend these simulations to the more physical self-avoiding case is of much interest, albeit very challenging.

Fluid membranes present another challenge to numerical simulations. They are expected always to crumple on long length-scales, yet computer simulations suggest a possible transition to a flat phase. However, these results where obtained is simulations of systems of modest size; revisiting fluid membranes now that better algorithms and faster computers are available would be an interesting project. 


\section{References}

[1] C. Rovelli, Strings, loops and others: a critical survey of the present approaches to quantum gravity, (gr-qc/9803024).

[2] S. Weinberg, in General Relativity. An Einstein centenary survey (1979), S.W. Hawking and W. Israel, eds. Cambridge University Press, p. 179.

[3] H. Kawai and M. Ninomiya, Nucl. Phys. B 336 (1990) 115;

H. Kawai, Y. Kitazawa and M. Ninomiya, ibid 404 (1994) 684.

[4] T. Regge, Il Nouovo Cimento 19 (1961) 558.

[5] M. Rocek and R. Williams, Phys. Lett. B 104 (1981) 31.

[6] D. Weingarten, Nucl. Phys. B 210 (1982) 229.

[7] R.M. Williams, Nucl. Phys. B 57 (Proc. Suppl.) (1997) 73.

[8] F. David, Simplicial quantum gravity and random lattices, (hep-th/9303127), Lectures given at Les Houches Summer School, Session LVII, (Les Houches, France, 1992).

[9] J. Ambjørn, B. Durhuus and T. Jónsson, Quantum Geometry: A statistical field theory approach, (Cambridge Univ. Press, 1997).

[10] C. Holm and W. Janke, Phys. Lett. B 335 (1994) 143;

W. Bock and J.C. Vink, Nucl. Phys. B 438 (1995) 320.

[11] M. Gross and S. Varsted, Nuc. Phys. B 378 (1992) 367.

[12] S. Jain and S.D. Mathur, Phys. Lett. B 286 (1992) 239;

J. Ambjørn, S. Jain and G. Thorleifsson, ibid 307 (1993) 34;

J. Ambjørn and G. Thorleifsson, ibid 323 (1994) 7.

[13] S. Catterall, G. Thorleifsson, M. Bowick and V. John, Phys. Lett. B 354 (1995) 56;

J. Ambjørn, J. Jurkiewicz and Y. Watabiki, Nucl. Phys. B 454 (1995) 313.

[14] T. Hotta, T. Izubuchi and J. Nishimura, Prog. Theor. Phys. 94 (1995) 263;

S. Catterall, G.Thorleifsson, J. Kogut and R. Renken, Nucl. Phys. B 468 (1996) 263;

S. Catterall, R. Renken and J. Kogut, Phys. Lett. B. 416 (1998) 274.

[15] J. Ambjørn, D.V. Boulatov, A. Krzywicki, S. Varsted, Phys. Lett. B 276 (1992) 432; J. Ambjørn and S. Varsted, Nucl. Phys. B 373 (1992) 557.

[16] T. Hotta, T. Izubuchi and J. Nishimura, Nucl. Phys. B 63 (Proc. Suppl.) (1998) 63; Multicanonical simulation of 3-d dynamical triangulation model and a new phase structure, hep-lat/9802021.

[17] P. Bialas,, Z. Burda, A. Krzywicki and B. Petersson, Nucl. Phys. B 472 (1996) 293.

[18] J. Ambjørn, Z. Burda, J. Jurkiewicz, C.F. Kristjansen, Phys. Lett. B 297 (1992) 253; J. Ambjørn, J. Jurkiewicz and C.F. Kristjansen, Nucl. Phys. B 393 (1993) 601;

R. Renken, S. Catterall and J. Kogut, ibid 422 (1994) 677;

J. Ambjørn, J. Jurkiewicz, S. Bilke, Z. Burda and B. Petersson, Mod. Phys. Lett. A 9 (1994) 2527. 
[19] B. Brügmann, Phys. Rev. D 47 (1993) 3330;

B. Brügmann and E. Marinari, Phys. Rev. Lett. 70 (1993) 1908

[20] R. Renken, S. Catterall and J. Kogut, Nucl. Phys. B 523 (1998) 552.

[21] S. Bilke, Z. Burda, A. Krzywicki, B. Petersson, J. Tabaczek and G. Thorleifsson, Phys. Lett. B 418 (1998) 266; ibid 432 (1998) 279.

[22] Cates, Europhys. Lett. 8 (1988) 719;

A. Krzywicki, Phys. Rev. D 41 (1990) 671.

[23] I. Antoniadis and E. Mottola, Phys. Rev. D 45 (1992) 2013;

I. Antoniadis, P.O. Mazur and E. Mottola, Nucl. Phys. B 388 (1992) 627; Phys. Lett. B 394 (1997) 49.

[24] F. David, Nucl. Phys. B 257 (1985) 543;

F. David, J. Jurkiewicz, A. Krzywicki, and B. Petersson, ibid. 290 (1987) 218.

[25] S. Bilke, Z. Burda, A. Krzywicki, B. Petersson, K. Petrov, J. Tabaczek and G. Thorleifsson, The strong-coupling expansion in simplicial quantum gravity, (hep-lat/9809088), these proceedings.

[26] P. Bialas, Z. Burda, B. Petersson, J. Tabaczek, Nucl. Phys. B 495 (1997) 463;

P. Bialas, Z. Burda and D. Johnston, ibid 493 (1997) 505;

P. Bialas and Z. Burda, Phys. Lett. B 416 (1998) 281.

[27] P. Bialas, Z. Burda and D. Johnston, Phase diagram of the mean-field model of simplicial gravity, (gr-qc/9808011).

[28] E. Brezin, C. Itzykson, G. Parisi, J.B. Zuber, Commun. Math. Phys. 59 (1978) 35.

[29] J. Ambjørn, G. Thorleifsson and M. Wexler, Nucl. Phys. B 439(1995) 187.

[30] G. Thorleifsson, Three dimensional simplicial gravity and degenerate triangulations, (hep-lat/9807026).

[31] S. Bilke and G. Thorleifsson, Simulating $4 D$ simplicial quantum gravity using degenerate triangulations, (hep-lat/9809106), these proceedings.

[32] S. Catterall, J. Kogut and R. Renken, Phys. Rev. Lett. 72 (1994) 4062;

J. Ambjørn and J. Jurkiewicz, Phys. Lett. B 335 (1994) 355; B. Brugmann and

E. Marinari, ibid 349 (1995) 35.;

S. Catterall, J. Kogut, R. Renken and G. Thorleifsson, ibid 366 (1996) 72.

[33] Statistical mechanics of membranes and surfaces, eds. D.R. Nelson, T. Piran and S. Weinberg, (World Scientific, Singapore, 1989).

[34] Fluctuating geometries in statistical mechanics and field theory, eds. P. Ginsparg, F. David and J. Zinn-Justin, (North Holland, Amsterdam, 1996).

[35] M. Bowick, S. Catterall, M. Falcioni, G. Thorleifsson and K. Anagnostopoulos, J. Phys. I France 6 (1996) 1321.

[36] H. Kleinert, Phys. Lett. A 114 (1986) 263;

D. Forster, ibid 114 (1986) 115. 
[37] J. Ambjørn, A. Irback, J. Jurkiewicz, B. Petersson, Nucl. Phys. B 393 (1993) 571;

M. Bowick, P. Coddington, L. Han and G. Harris, ibid 394 (1993) 791.

[38] L. Radzihovsky and J. Toner, Phys. Rev. Lett. 75 (1995) 4752;

Phys. Rev. E 57 (1998) 1832.

[39] M. Bowick, M. Falcioni and G. Thorleifsson, Phys. Rev. Lett. 79 (1997) 885.

[40] G. Thorleifsson and M. Falcioni, Comp. Phys. Comm. 109 (1998) 161.

[41] M. Bowick and E. Guitter, Phys. Rev. E 56 (1997) 7023.

[42] M. Bowick and A. Travesset, The tubular phase of self-avoiding anisotropic crystalline membrane, cond-mat/9808214); New analytical results on anisotropic membranes, (hep-lat/9809110), these proceedings.

[43] M. Bowick, S. Catterall, S. Warner, G. Thorleifsson and M. Falcioni, Anisotropic membranes, (hep-lat/9809112), these proceedings. 